\title{
High Reliable And Intelligent Primary Medical Information System
}

\author{
Bo Liu ${ }^{a}$, Gang Shi ${ }^{\text {* }}$, Li Huyang ${ }^{\mathrm{c}}$ \\ Shenyang Institute of Automation Chinese Academy of Sciences, China \\ aliubo@sia.cn, bsg0105@sia.cn, clihuyang@sia.cn
}

Keywords: primary medical; intelligent temperature control; infrared ear temperature; battery capability monitoring

\begin{abstract}
According to the medical pattern and problems of our country, this paper researches and develops a kind of high reliable and intelligent primary medical system, which has strong adaptability to the environment and long duration. It can also replace community medical practitioners to do some necessary works, such as recognition and extraction of identity information, acquisition control and data processing of health information, generating and printing test report. Thus, the current situation of primary medical resources shortage can be alleviated.
\end{abstract}

\section{Introduction}

Nowadays, the medical pattern of our country is "inverted pyramid". The problem of uneven distribution of medical resources is existing, which causes the central hospitals have excessive pressure with long queue length, long waiting time, and short treatment time. But because of the lack of medical resources, primary hospitals are unable to meet the daily basic medical demands of people.

According to the above problems, the high reliable and intelligent primary medical system, which is researched and developed by the means of information technology, is needed. It can implement decentralizing the medical resources of the central hospitals. The community medical practitioners can use these primary medical systems to offer their daily services in the primary hospitals, and offer the necessary primary medical services to the community residents at any time, such as health insurance and follow-up screening. In the first aid and other special circumstances, the community medical practitioners can carry out the rapid medical examination and diagnosis with these primary medical systems on the spot, so that the quick response ability and quality of pre-hospital emergency treatment are improved, which not only is related to whether save the lives of patients or not, but also has the decisive influence with the prognosis.

In order to give full play to the advantages of the high reliable and intelligent primary medical system, based on the above application scenes, the following characteristic in three respects are needed to be considered during the design plan of the system:

- The system should have a high degree of intelligence. It can replace the community medical practitioners to do some necessary work, so that the community medical practitioners can focus on the medical treatment of patients and the efficiency of medical treatment can be improved.

- The system should have strong adaptability to the environment. Through real-time monitoring the system environment temperature and controlling the heating and radiating devices, it can adapt to the working environments with the different temperatures.

- The system should have long duration. Because the primary medical systems may be used outdoors, ensuring a long working time is equal to increasing the patients' medical treatment time and the probability of survival and rehabilitation of patients.

\section{The Overall Architecture of the System}

According to the above design requirements of the system, this paper researches and develops a kind of high reliable and intelligent primary medical system, which overall architecture is as shown in figure 1 . The system can implement the rapid measurement of body temperature based on infrared ear thermometer principle, and implement many functions, such as recognition and extraction of identity 
information, acquisition control and data processing of health information, generating and printing the test report, system state configuration and manual operating, and so on. At the same time, the system can also real-time monitor the environment temperature of the system, control the heating and radiating devices, real-time monitor the battery capability of the system, and have power alarm function.

\begin{tabular}{|c|c|c|c|}
\hline $\begin{array}{c}\text { Control } \\
\text { Level }\end{array}$ & $\begin{array}{l}\text { Heating Radiating } \\
\text { Device Control }\end{array}$ & $\begin{array}{c}\text { Fault Detection } \\
\text { Alarm }\end{array}$ & $\begin{array}{c}\text { System State } \\
\text { Configuration }\end{array}$ \\
\hline $\begin{array}{l}\text { Perceive } \\
\text { Level }\end{array}$ & $\begin{array}{l}\text { Temperature } \\
\text { Monitoring }\end{array}$ & $\begin{array}{c}\text { Battery Capability } \\
\text { Monitoring }\end{array}$ & $\begin{array}{l}\text { Switch Input } \\
\text { Recognition }\end{array}$ \\
\hline $\begin{array}{l}\text { Interface } \\
\text { Level }\end{array}$ & $\begin{array}{l}\text { Health Information } \\
\text { Collection }\end{array}$ & $\begin{array}{c}\text { Identity } \\
\text { Auto-Recognition }\end{array}$ & Printer Output \\
\hline
\end{tabular}

Figure 1. The overall architecture of the system

In the aspect of intelligence, the system can control radiofrequency card to recognize the patients' identification card, automatically extract the patients' identity information and entry them to the internal main control unit of the system. The system establishes the health records in corresponding with the patients on the basis of identity information, so that the community medical practitioners can collect and view the health status information of the patients. In addition, the system can not only control health information collection module to real-time collect the health information of the patients, and real-time transmit the collected health information to the internal main control unit of the system to be analyzed, calculated and handled, but also driver the embedded thermal printer to print the health records in the format of test report, which contains the identification information and health information of the patients.

In the aspect of adaptability to the environment, the system can real-time monitor the system environment temperature. When the temperature is lower than the lower threshold, the ceramic heating sheets are automatically switched on for heating the system inside. When the temperature is higher than the upper threshold, the silent fan is automatically switched on for radiating the system inside. Moreover, the system real-time monitors the running state of the fan. If some fault conditions are occur, for example the fan is locked rotor, a warning signal of the system is immediately sent. According to the different application scenes and climate conditions, the community medical practitioners can set the upper and lower temperature threshold, in order to improve the system adaptability to the environment.

In the aspect of duration, the system can real-time monitor the battery capability of the system, and prompt the community medical practitioners through the operation interface. When the battery capability is not enough, a warning signal of the system is immediately sent. After a delay of more than waiting time, the system enters the shutdown state. According to the situation that the community medical practitioners forget to shutdown at the end of the use, the system can automatically recognize the closing cover signal. After a delay of more than waiting time, the system enters the standby state.

\section{Hardware Circuit Design of the System}

In this paper, the schematic diagram of the hardware circuit of the high reliable and intelligent primary medical system mainly contains intelligent temperature control module, fault detection module and power management module.

A. Intelligent Temperature Control Module

The intelligent temperature control module applies two kinds of temperature sensors, temperature control switch and DS18B20. According to the mutative temperature of the working environment, physical deformation occurs in the internal of the temperature control switch, resulting in conduction or break action. The system applies the temperature control switch to cope with the extreme temperature condition before the system is working. When the extreme low environment temperature causes the system doesn't work, through the temperature control switch converting from open state to 
closed state, the ceramic heating sheets are automatically switched on for heating the system inside. When the temperature of the system is higher than $0{ }^{\circ} \mathrm{C}$, the system can be started normally. Through the temperature control switch converting from closed state to open state, the ceramic heating sheets are automatically stopped to heat. When the extreme high environment temperature causes the system doesn't work, through the temperature control switch converting from open state to closed state, the fan is automatically switched on for radiating the system inside. When the temperature of the system is lower than $50{ }^{\circ} \mathrm{C}$, the system can be started normally. Through the temperature control switch converting from closed state to open state, the fan is automatically stopped to radiate.

After started, the system applies the DS18B20 sensor to real-time monitor the system environment temperature. According to the relationship between the system temperature and the temperature threshold, through controlling ceramic heating sheets and fan, the accurate maintain of the system environment temperature is implemented.

\section{B. Fault Detection Module}

The fault detection module consists of the voltage conversion circuit and the continuous triggered monostable circuit. The continuous triggered monostable circuit consists of 555 timer chip, transistor and other electronic components [2].

When the Trigger signal has a falling edge, the 555 timer is from the steady state with low level output into the temporary steady state with high level output. During the temporary steady state, if the Trigger signal still has a falling edge, the 555 timer will continue the temporary steady state with high level output. Otherwise, after the temporary steady state, 555 timer will restore the steady state with low level output. The fan feedback signal is the pulse signal. When the fan is operating normally, the duty ratio of the pulse signal is fixed. The falling edge has already appeared in the 555 timer transient period, so the 555 timer has already been the temporary steady state with high level output, as shown in figure 2. When the fan rotation speed reduces with error, the duty ratio of the pulse signal is increasing. During the temporary steady state, the fan feedback signal is already high without the falling edge, so the 555 timer will restore the steady state with low level output after the temporary steady state, that is the fault alarm signal.

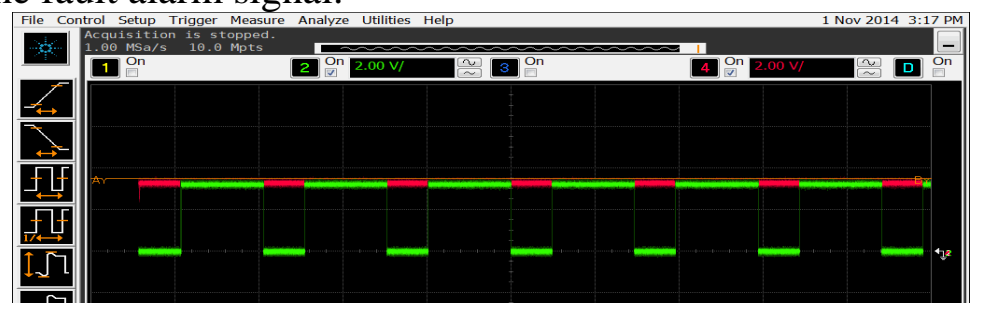

Figure 2. The waveform when the fan is operating normally

C. Power Management Module

The power management module applies the power conversion chip TPS54331, which converts the adapter input $24 \mathrm{~V}$ voltage into the stable output voltage, and sufficiently supplies the internal modules of the system with the $3 \mathrm{~A}$ maximum continuous output current. At the same time, the power management module applies the battery charging management chip CN3704 to automatically manage charging the system battery. The CN3704 chip has two charging modes, constant current and constant voltage [3]. When the battery voltage is lower than $16.8 \mathrm{~V}$, the power management module enters into the constant current charging mode. At this time, the charging current is determined by the internal reference voltage $200 \mathrm{mV}$ and an external resistor $R_{C S}$, that is, the charging current is $200 \mathrm{mV} / \mathrm{R}_{\mathrm{Cs}}$. When the battery voltage rises to almost $16.8 \mathrm{~V}$, the power management module enters into the constant voltage charging mode as the charging current is reduced gradually. When the charging current is reduced to the value, which is set by the resistance of the chip pin EOC, the charging of the battery is finished.

\section{Software Program Design of the System}

In this paper, the high reliable and intelligent primary medical system implements the communication and control functions based on $\mu \mathrm{C} / \mathrm{OS}-\mathrm{II}$ operating system. $\mu \mathrm{C} / \mathrm{OS}$-II is a portable, 
curable, tailored, preemptive multi-task real-time operating system kernel. Many necessary system services are supplied on the kernel, such as semaphore, mailbox, event flag group, message queue, interrupt vector, and so on, for implementing the functions, such as task scheduling, task management, time management, memory management, communication and synchronization among tasks [4]. The high reliable and intelligent primary medical system includes three sub tasks:

- One sub task controls the acquisition of health information, and data processing and transmission of health information collected.

- Another sub task real-time monitors the system battery capability, according to the relationship between the battery output voltage and the battery capability.

- The other sub task recognizes and drivers the system peripherals, such as switches, radiofrequency module, and embedded thermal printer.

\section{A. Infrared Ear Temperature Detection}

The hypothalamus is an important brain organ, which is nearest with ear and controls body temperature. As the average temperature of the deep body changes, the ear temperature is also rapidly changing. And inner ear is a closed area, affected little by the external factors, so ear temperature is closest to the body temperature [5]. Trough measuring the infrared radiation of the ear canal and tympanic membrane, the infrared ear thermometer measures temperature, radiation energy density of which is proportional to temperature. The system applies 90615 sensor of Melexis company to develop infrared ear temperature detection module based on SMBUS communication protocol, which contains reading sensor address, write object emissivity, read object temperature, and so on.

As found in the actual detection, when the detection probe is measuring temperature into the ear canal, thermal shock phenomenon will occur. So temperature compensation is necessary, which formula is as in (1).

$$
\mathrm{To}_{\text {comp }}=\mathrm{To}_{\text {meas }}-\left(\mathrm{a}+\left(\mathrm{To}_{\text {meas }}-\mathrm{Ta}\right) \times \mathrm{b}\right) .
$$

Among (1), Tocomp is the object temperature after the compensation. Tomeas is the object temperature before the compensation. Ta is the environment temperature. $\mathrm{a}$ and $\mathrm{b}$ are the temperature compensation coefficients, which values are determined by the experiment.

The experiment method of the temperature compensation coefficients is as follow. The temperature of the ear blackbody must be set fixed to simulate the human ear canal temperature and the detection probe enters into the ear blackbody. The frequency of reading temperature is set 4 times per second. In the experiment, the detection probe enters into the ear blackbody for 10 seconds, that means reading 40 sets of temperature data, and pulls out from the ear blackbody for 10 seconds. Successively, 600 sets of temperature data are necessary for every experiment. According to (2), through constantly test the temperature compensations, the object temperature after the compensation is made in the range of the ear blackbody temperature $\pm 0.2^{\circ} \mathrm{C}$, as shown in figure 3 , that means the temperature compensation coefficients is suitable to the detection probe of this test. Though experimenting with 9000 sets of temperature data, which are read form the experiments of five different environment temperature and three different blackbody temperature, this paper obtains the temperature compensation coefficients suitable to the detection probe of the system.

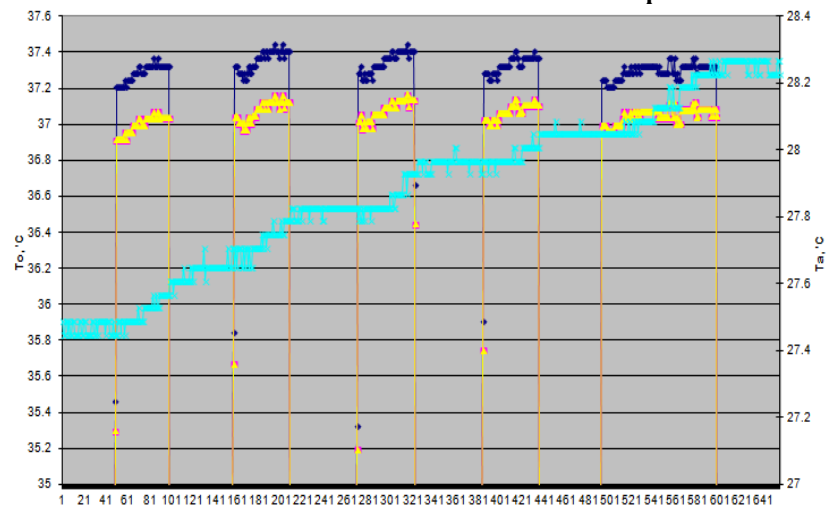

Figure 3. Data analysis of infrared ear temperature compensation experiment 


\section{B. Battery Capability Monitoring}

The system applies the voltage follower circuit to monitor the battery output voltage, and applies 12 bit ADC module to convert battery output voltage from analog signal to digital signal. According to the relationship between the battery output voltage and the battery capability, the battery capability is calculated.

The derivation process of the relationship between the battery output voltage and the battery capability is as follow. First of all, through the experiment, each discrete battery output voltage and current are recorded at intervals of 5 seconds. Through calculating the integration of the output current at the time, the consumed battery capability is known. So the relationship between each discrete battery output voltage and battery capability can be derived. Then the curve fitting of the relationship in MATLAB software is as shown in figure 4.

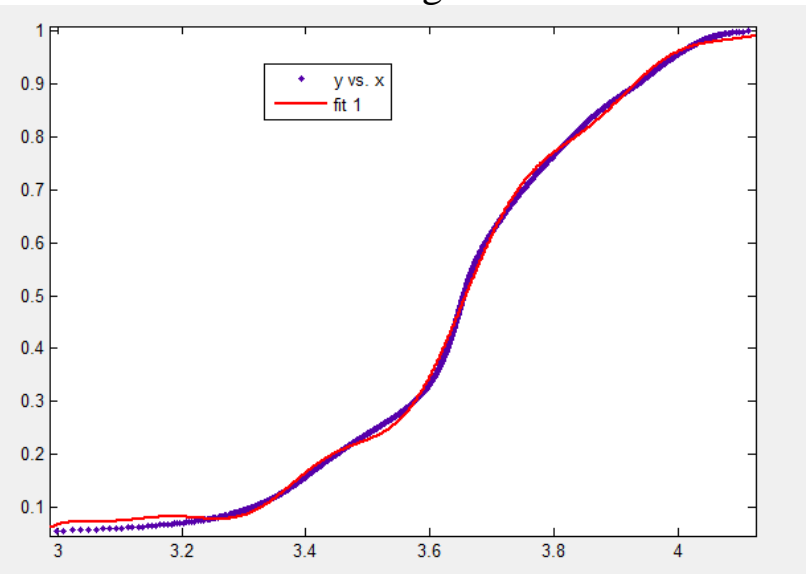

Figure 4 . The relationship between battery output voltage and battery capability

Among figure 4, the blue curve is the relationship curve between battery output voltage and battery capability by the connection of the discrete record points, which $\mathrm{x}$-axis is the battery output voltage and $y$-axis is the battery capability. The red curve is the approximate curve fitted by MATLAB software. Thus, the equation between battery output voltage and battery capability is obtained as in (2).

$$
\mathrm{y}=\mathrm{a} 1 \sin (\mathrm{b} 1 \mathrm{x}+\mathrm{c} 1)+\ldots+\mathrm{a} 4 \sin (\mathrm{b} 4 \mathrm{x}+\mathrm{c} 4)
$$

Among (2), $\mathrm{y}$ is the battery capability. $\mathrm{x}$ is the battery output voltage. $\mathrm{a} 1$ is $6.153, \mathrm{~b} 1$ is $0.1537, \mathrm{c} 1$ is 5.805 . a 2 is $0.1472, \mathrm{~b} 2$ is $6.671, \mathrm{c} 2$ is 5.687 . a 3 is $0.02899, \mathrm{~b} 3$ is $16.72, \mathrm{c} 3 \mathrm{is}-23.53$. a 4 is 0.01885 , b4 is 24.05 , c4 is 24.93 .

C. Recognizing and Drivering Peripherals

The program flow to recognize and driver peripherals is as shown in figure 5 .

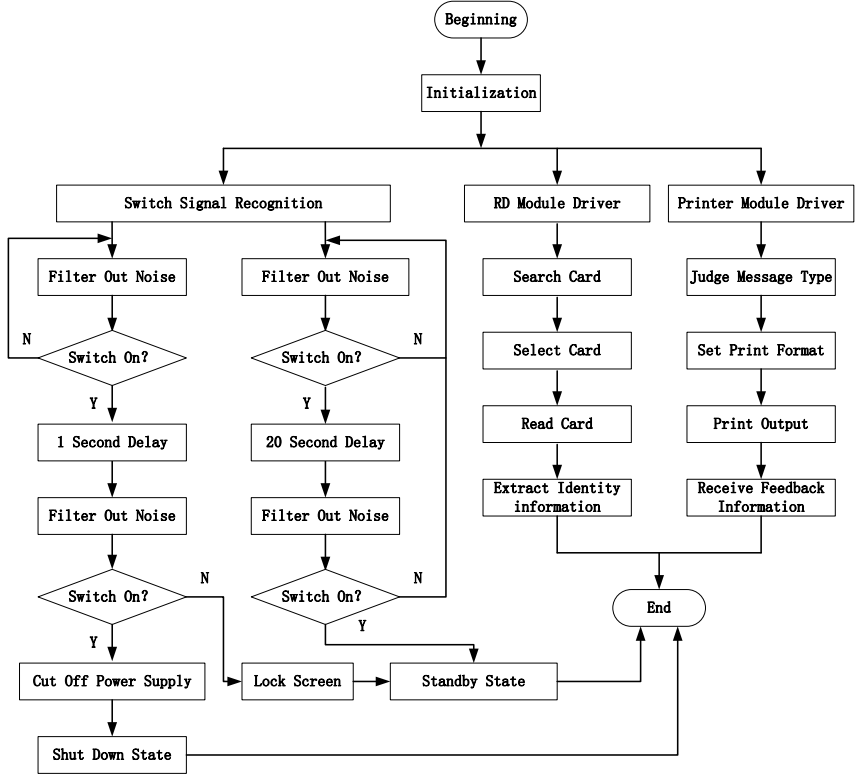

Figure 5. The program flow to recognize and driver peripherals 
The system real-time monitors the switch signals and eliminates the influence of switch jitter noise signal by filtering algorithm. If the button is pressed for longer than 1 second, the system considers that is a shutdown signal and cuts off the power supply of internal modules to enter the shutdown state. If the button is pressed for less than 1 second, the system considers that is a lock-screen signal and cuts off the power supply of the display screen to enter the standby state. When the closing cover signal is active, the system considers that community medical practitioners forget to shutdown at the end of use. After a delay of more than waiting time, the system enters the standby state. At the same time, through USART based on modules' communication protocols, the system can driver radiofrequency recognizing and output printing modules to implement the functions, such as recognition and extraction of identity information, generating and printing the test report, and so on.

\section{Conclusions}

From the above descriptions, the high reliable and intelligent primary medical system researched and developed by this paper, has strong adaptability to the environment by the intelligent temperature control and long duration by the power management. Through the detection of infrared ear temperature and the driver of the peripherals it can also replace community medical practitioners to do some necessary works. Thus, the current situation of primary medical resources shortage can be alleviated.

\section{Acknowledgment}

This work is supported by the National Key Technology Research and Development Program of China under Grant No. 2015BAH09F03.

\section{References}

[1] ST Company, “STM32F103x8 STM32F103xB,” www.st.com, April 2009.

[2] Youqing Chen, "The principle, design and application of a 555 time base circuit," Publishing house of electronics industry, September 2007.

[3] Consonance Electronic Company, "The integrated circuit of charging management about 5A three lithium battery," www.consonance-elec.com.

[4] Jean J. Labrosse, “MicroC/OS-II The Real-Time Kernel,” Beihang University Press, May 2003.

[5] Jilin Wei, Haiyang $\mathrm{Wu}$, and Xuanbing Qiu, "Design of infrared ear thermometer based on MLX90615,” Ome Information, vol. 28, No. 6, pp. 35-38, June 2011. 